\title{
NUTRITION IN HIV: A REVIEW
}

\section{*L. M. Houtzager}

*Nutrition Development Division, Albion Street Centre, Sydney, Australia

\section{Correspondence:}

Louise M. Houtzager

Nutrition Development Division

Albion Street Centre

150-154 Albion Street

Surry Hills, NSW

Australia 2010

Email: louise.houtzager@sesiahs.health.nsw.gov.au

\begin{abstract}
Knowledge about the relationship between the human immunodeficiency virus (HIV), nutritional status, treatment, food and nutrition interventions continues to accumulate. This article provides an overview of the main nutrition related issues for people living with HIV (PLHIV) and a review of the potential benefits of nutrition interventions for people affected by HIV.

Nutrition plays a vital role in the immune system of all people, including (PLHIV). Good nutrition strengthens the immune system, while HIV infection and poor nutrition have a cumulative effect in damaging it. PLHIV are more vulnerable to malnutrition than the general population and nutritional status is a good predictor of their mortality risk. Malnutrition in PLHIV often occurs in a background of poverty and lack of access to food.
\end{abstract}

It is not always possible to identify one single cause as the main contributor to declining nutritional status or malnutrition in HIV. Inadequate food intake, increased requirements and malabsorption are the main reasons for weight loss in PLHIV. Asymptomatic adults with HIV infection have a $10 \%$ higher energy requirement and symptomatic PLHIV have $20-30 \%$ higher energy requirements than the general population. Kilojoule/kilocalorie requirements increase by $50-100 \%$ in children experiencing weight loss. Evidence for increasing protein and micronutrient intake in healthy PLHIV is inadequate.

Nutritional education should be an essential component of HIV care and treatment, as it can help PLHIV cope with symptoms of disease, prevent weight loss and manage side effects of medication. In resource limited settings, food support programs may be required in addition to nutrition support to optimise nutritional status and health outcomes in PLHIV who are food insecure.

Key words: Nutrition, HIV, weight loss, malnutrition, diarrhoea, macronutrients, micronutrients, food security

\section{INTRODUCTION}

The World Health Organisation (WHO) and UNAIDS estimated that at the end of 2007, there were 33 million people living with HIV infection (PLHIV) worldwide, with $67 \%$ of these living in Sub-Saharan Africa ${ }^{1 .}$ In Benin it is estimated that 64000 people were living with HIV in 2007. Access to antiretroviral therapy (ART) is increasing in Benin, however 
coverage is only estimated to be about $50 \%$ or 9800 of 20000 PLHIV requiring treatment ${ }^{2}$.

Despite major advances in the treatment and survival of PLHIV, weight loss and wasting remain common problems ${ }^{3}$. Poor nutritional status in PLHIV is associated with disease progression, increased morbidity, and reduced survival ${ }^{4,5}$, even when ART is available ${ }^{3}$.

Incorporating nutrition interventions into HIV care and treatment programs can help improve health outcomes for PLHIV ${ }^{6}$. Nutrition is an integral part of HIV care at any stage of disease. Nutrition and food security interventions have the potential to keep PLHIV well for longer and improve their response to $\mathrm{ART}^{7}$. This article provides an overview of the main nutrition related issues for PLHIV and the potential benefits of nutrition interventions for people affected by HIV.

\section{THE RELATIONSHIP BETWEEN HIV AND NUTRITION}

The impact of HIV infection on nutrition was identified early in the epidemic, with wasting one of the most visible signs of malnutrition in patients who progress to acquired immune deficiency syndrome (AIDS) ${ }^{8-10}$. HIV infection and poor nutritional status are interlinked. Malnutrition impairs immune function and reduces the body's resistance to infection ${ }^{11}$. HIV and malnutrition have a cumulative effect in damaging the immune system and worsening nutritional status. PLHIV are more vulnerable to malnutrition than the general population. Conversely people with malnutrition, related to food insecurity, may increase their risk of contracting HIV infection by engaging in sex work to generate income for food ${ }^{12}$.

Several nutritional indicators including weight $\operatorname{loss}^{13,6}$, low body weight/muscle mass ${ }^{14-16}$, anaemia ${ }^{17}$, and low serum albumin ${ }^{18}$ have been associated with shorter duration of survival in PLHIV. A large study in West Africa found that Body Mass Index (BMI) following diagnosis of HIV could predict death to a similar extent as CD4 cell count. PLHIV with lower body weights had an increased risk of death $^{19}$.

\section{COMMON FACTORS AFFECTING NUTRITIONAL STATUS IN PLHIV}

It is not always possible to identify one single cause as the main contributor to declining nutritional status or malnutrition in HIV. Primary and secondary malnutrition may exist together in PLHIV worsening the effect on health. Primary malnutrition is caused by inadequate consumption of food and essential nutrients due to: food not being available or accessible; or poor eating habits. Secondary malnutrition results from infection or disease, leading to increased energy expenditure, malabsorption or altered utilisation of nutrients.

\section{Inadequate food intake}

Loss of appetite leading to reduced food intake (and nutrients) is one of the main reasons why people lose weight with HIV infection ${ }^{20}$. Reduction in dietary intake may lead to growth failure in HIV-positive children ${ }^{21}$ and wasting in HIV-positive adults ${ }^{22}$. Inadequate food intake prevents recovery from malnutrition.

Inadequate food intake is caused by many different factors. In addition to lack of food, the desire to eat may be reduced in PLHIV by various factors, including but not limited to:

- Oral candidiasis (OR), mouth or gastrointestinal tract ulceration/ irritation making eating and swallowing uncomfortable. 
- Infections resulting in reduced appetite, nausea, vomiting and/or diarrhoea.

- Metabolic effects of malnutrition, diarrhoea and drugs.

- Psychosocial factors including depression and lack of emotional support.

- Antiretroviral side effects such as nausea and diarrhoea

\section{Malabsorption}

Intestinal malabsorption leading to energy $(\mathrm{KJ} / \mathrm{Kcal})$ and nutrient losses is common among $\mathrm{PLHIV}^{23,24}$. Chronic weight loss and malabsorption in HIV is often related to gastrointestinal diseases which cause severe diarrhoea $^{25,26}$ or HIV enteropathy. PLHIV with more severe malabsorption have lower body weights $^{27}$.

\section{Increased energy expenditure}

PLHIV have a resting metabolic rate (RMR) that is around $10 \%$ higher compared to HIV negative adults, and this $20-30 \%$ higher when they have secondary or opportunistic infections ${ }^{28-}$ 32. Increased energy expenditure will often lead to weight loss in PLHIV. This weight loss may occur despite an individual maintaining their usual food intake after acquiring HIV, and during all stages of the disease.

\section{Psychosocial factors}

Several factors amplify and complicate malnutrition and HIV including mental illness, personal beliefs, poverty, and food insecurity. Food insecurity occurs more often in PLHIV as the majority of HIV infections occur in the most productive section of the population, which is amongst adults aged $15-49^{33}$. As PLHIV become unwell, work participation and income generating capacity decreases, leading to lower household incomes and less money available for food particularly when medical costs increase at the same time $^{34,35}$. Declining health and mobility can also impact on an individual's ability to access a reliable food supply, depending on the availability of social supports.

Personal religious and cultural beliefs may prohibit the consumption of certain foods and influence decision making around food and treatments ${ }^{6}$. Beliefs about the curative powers of certain foods and herbal therapies may prevent achievement of optimal nutritional status, either by refusal to take ART when necessary or replacing nutritious foods with nutritionally inadequate foods or supplements.

\section{IMPACT OF ART ON DIETARY INTAKE AND NUTRITIONAL STATUS}

One of the most important observations about the interaction between ART and nutritional status is that initiating ART often leads to a reversal of symptoms caused by HIV such as malnutrition and loss of body mass (including muscle mass). Increased appetite, improved food intake and reduced viral load improve nutritional status. This improvement is associated with a reduction in morbidity and mortality from HIV related causes ${ }^{36}$.

There are a number of food interactions that influence not only the absorption and utilisation of ART but also impact on digestion, absorption and assimilation ${ }^{37}$. It is also known that certain antiretroviral therapies can produce a range of side effects and metabolic complications that have a significant impact on health and wellbeing $^{37}$.

Some of the more common side effects include diarrhoea, loss of appetite, bloating, and nausea and unexplained weight change.

There is a growing amount of evidence about the long term 
complications of ART in a significant proportion of adults, children and infants living with HIV. These metabolic complications include disorders such as lipodystrophy, dyslipidaemia, insulin resistance, abnormalities in glucose tolerance, lactic acidosis, mitochondrial toxicity, and bone demineralisation. It appears that these complications may be related to particular drugs. These side effects may have serious consequence in terms of adherence with ART, increased risk of chronic diseases including cardiovascular disease and diabetes, and reduced quality of life. These metabolic effects not only impact on the health and wellbeing of PLHIV but may necessitate a shift to another ART regime.

Despite advances in the knowledge about interactions between ART and nutritional status, many questions remain unanswered. The consequences and efficacy of ART among individuals with pre-existing (primary) malnutrition are unclear. The effects of underlying malnutrition on the absorption and metabolism of all or individual ART medication are not fully understood ${ }^{37}$. It is also unclear whether nutritional supplementation can prevent or reduce the occurrence of complications or side effects due to ART.

Among infants and children, the impact of acute or chronic severe malnutrition on immune function and response to ART, including experience of side effects is not well understood. Further research and monitoring is also required to elucidate the impact ART has on growth among infants and children, particularly from resource poor settings.

In many parts of the world, supplementation using herbal and alternative therapies is a common practice for many PLHIV. Although the effects of these supplements are becoming clearer there is still much that needs to be learned about the interactions between ART medication and various traditional therapies ${ }^{37}$.

\section{NUTRITION SUPPORT AND ART}

Although conclusive evidence is lacking there are a small number of studies suggesting that food and nutrition support may improve treatment outcomes. Most studies on the effectiveness of ART have been done in populations where primary malnutrition is not endemic. The effect of malnutrition on the absorption and metabolism of antiretroviral therapies in areas where food is scarce requires further research.

A study of PLHIV in Singapore examined the impact of malnutrition at the time of commencing $\mathrm{ART}^{38}$. Stage of disease, number of previous opportunistic infections, CD4 count and Viral Load were all significantly associated with Body Mass Index (BMI)/ weight. The authors of this study concluded that malnutrition was significantly associated with reduced survival in patients commencing ART. Another study published in the United States found that micronutrient supplementation significantly increased CD4 count among PLHIV on $\mathrm{ART}^{39}$.

Some PLHIV report a concern that starting antiretroviral therapy may result in increased appetite; this may be an important factor in the decision to initiate treatment for food insecure PLHIV. A study in Zambia found that providing food support to food insecure patients increased ART adherence by $40 \%$ and increased weight during the first 6 months of treatment ${ }^{40}$. Interventions using supplements or food at time of ART commencement may improve treatment outcomes. 


\section{BENEFITS OF NUTRITION INTERVENTIONS}

Nutrition education can assist PLHIV in various settings access and make nutritious, affordable and culturally appropriate food choices. Dietary counselling, especially when provided early have been demonstrated to improve the body weight of PLHIV ${ }^{41,42}$. Anecdotally dietitians and other health care workers report that providing nutritional support and dietary counselling to minimise weight loss or treat malnutrition, appears to improve the physical and psychological health of PLHIV. However, scientific evidence for the effectiveness of dietary interventions is limited.

\section{Symptom management}

Symptoms of HIV infection or related illnesses contribute to both primary and secondary malnutrition in PLHIV. Symptoms may impact on nutritional status by reducing the desire to eat, changing the types of foods consumed, reducing the amount of food eaten, and by affecting the absorption and utilisation of nutrients.

Diarrhoea is one of the most common symptoms experienced by PLHIV. Diarrhoea is the passage of 3 or more loose or liquid stools per day, or more frequently than is normal for the individual ${ }^{43}$. The causes of diarrhoea will vary depending on an individual's immune status. Parasites, bacteria and viruses may account for up to $80 \%$ of diarrhoea experienced by PLHIV. Medications, including ART, may also cause or exacerbate diarrhoea in PLHIV.

Diarrhoea is a symptom. When possible, the underlying cause of diarrhoea should be identified and treated. In some instances symptomatic treatment alone may be adequate or all that is available but in most cases investigation is preferable to determine the cause prior to medical intervention. Symptom control strategies include dietary modification, fluid and electrolyte replacement (eg an oral rehydration solution of $1 / 2$ tsp of salt, $8 \mathrm{tsp}$ of sugar to $1 \mathrm{~L}$ of boiled water) and anti-diarrhoeals. These are often necessary in addition to further medical interventions, particularly in cases of moderate to severe diarrhoea in PLHIV.

One clinical trial, which assessed the effect of dietary modifications on HIV related diarrhoea using normal foods (ie non supplemental) showed a significant reduction in stool frequency and consistency. The intervention diet included $50 \%$ reduction in intake of fat, lactose and insoluble fibre, $50 \%$ increase in soluble fibre and the elimination of caffeine. These dietary modifications resulted in a $28 \%$ reduction in stool frequency in the treatment groups vs $15 \%$ in the controls. Stool consistency improved $20 \%$ in the treatment group vs $8 \%$ in the controls ${ }^{44}$.

Oral health conditions also affect up to $90 \%$ of PLHIV at some time during the course of their disease $^{44}$. Oral lesions may be associated with a variety of infectious, neoplastic or inflammatory conditions.

Oral candidiasis (OC) occurs frequently in PLHIV and usually presents as white or yellow plaques on the roof of the mouth, tongue and inside cheeks. The incidence and severity of OC increases as the CD4 count decreases. Oropharyngeal candidiaisis is more common in patients with CD4 counts $<200$ cells $/ \mu$ l. Mouth infections including candidiasis can alter taste and reduce appetite.

Painful lesions in the mouth, throat and oesophagus contribute significantly to the reduction in food and liquid intake in PLHIV and warrant 
aggressive investigation and management where possible.

Treatment of candidiasis in PLHIV includes topical agents where oral disease is mild (eg nystatin emulsion, Miconazole gel or amphotricin lozenges), and systemic treatment with Fluconazole or Itraconazole for more severe disease.

There are no particular foods which affect recovery from candidiasis or ulceration although ulcerated lesions are usually more sensitive to salty, spicy and acidic agents. Eating a healthy diet is important for good health, though often food intake is reduced due to mouth soreness or painful swallowing. Modifying the texture and type of foods can help ensure adequate food intake.

The goals of dietary management for mouth pain, taste changes, chewing and swallowing difficulties include:

- to assist patients in maintaining a safe and comfortable food intake,

- prevent development of further oral health problems;

- maintain nutritional status and prevent weight loss

Other symptoms affecting dietary intake or requiring dietary intervention in PLHIV include: poor appetite, reflux; nausea and vomiting. Small frequent meals are advised for all and adequate re-hydration if vomiting present.

\section{Micronutrient supplementation in HIV}

Anaemia is a common problem for PLHIV and contributes to fatigue and malaise as well as increasing the risk of HIV disease progression.

There are a number of different causes of anemia in PLHIV including:
- Deficiency in iron, B12 or folate from inadequate intake or malabsorption

- Chronic illnesses such as TB and malaria

- HIV medications, particularly Zidovudine

- Increased iron needs eg pregnancy

- HIV infection per se

- Blood loss.

The best way to prevent anemia is by eating a wide variety of nutritious food. Iron absorption is influenced by the body's stores of iron. It is not just the amount of iron in the diet is important, but the amount that the body can absorb. Iron supplements may be beneficial in deficiency states but there are reports of deleterious effects in ongoing infections including malaria. Current guidelines for iron supplementation are unclear and a recent Cochrane review concluded that "the current clinical practice of iron supplementation in HIV-infected children is based on weak evidence comprising observational studies and expert opinions" and that high-quality RCTs of iron supplementation are urgently required, especially in areas with significant overlap of high prevalence of HIV, iron deficiency anaemia, and malaria ${ }^{46}$.

Some studies suggest a benefit in using micronutrient supplementation to slow HIV disease progression. A study in Tanzania found that women who received high dose multivitamin supplementation were less likely to have progression to advanced stages of HIV infection ${ }^{47}$. These findings were supported by a study of HIV positive males and females with advanced symptoms in Thailand which showed decreased rates of death in adults who received high dose micronutrient supplementation ${ }^{(48)}$. 
Interventions to improve micronutrients intake and nutritional status may contribute to a reduction of impact of the epidemic; however more research is needed before recommendations can be made. The relationship between micronutrient status, multivitamin use and slower disease progression has been documented in studies of PLHIV in the United States and Africa ${ }^{47-53}$. Vitamin supplementation in HIV infected women may improve growth in their infants and improve birth weights but may have adverse effects of PMTCT ${ }^{54}$. Zinc supplementation in HIV infected children was recently found to reduce morbidity caused by diarrhoea ${ }^{55}$. In addition regular megadoses of vitamin $A$ to HIV-positive children under 5 years of age have also been shown to reduce diarrhoeal morbidity ${ }^{56}$ and death rates ${ }^{57}$.

In adults, multivitamin supplementation has resulted in significantly lower viral loads, reduced incidence of complications such as oral thrush or gastrointestinal effects including nausea and diarrhoea in PLHIV in Africa ${ }^{47}$.

Despite the positive benefits reported with some micronutrient formulations, there is still insufficient evidence to recommend high dose supplements for all PLHIV and some studies have suggested harmful affects from vitamin and mineral supplementation. For example; maternal vitamin $\mathrm{A}$ and $\beta$-carotene supplements have been shown to significantly increase risk of mother-tochild transmission of HIV in randomized trials and can increase mortality in some children born to positive mothers ${ }^{58}$.

More evidence is required to establish optimal dietary and supplemental intake of vitamins and minerals to reduce progression of HIV to AIDS $^{59}$. Currently WHO does not recommend micronutrients above the level of recommended micronutrients for healthy non-HIV-infected persons of the same age, sex, and physical activity level ${ }^{60}$.

Conclusions from WHO's Consultation on Nutrition and HIV in $2005^{59}$ included:

- micronutrient status and intake may affect HIV transmission, progression and morbidity.

- some micronutrient supplements may be beneficial and others may have adverse effects,

- the same micronutrient supplement may be beneficial in some settings and have adverse effects in others.

In clinical practice, if food sources of vitamins and minerals are not available in adequate amounts, supplements may be recommended up to the recommended dietary intake level for the general population.

\section{Macronutrient requirements in HIV}

According to WHO energy requirements increase by $10 \%$ in asymptomatic HIV-infected adults ${ }^{4}$. For symptomatic PLHIV, energy requirements increase by $20-30 \%$ for adults and by $50-100 \%$ for children experiencing weight loss. Protein should provide 10 to $15 \%$ of daily energy intake, fat should provide at least $17 \%$ of energy intake, and carbohydrate up to $70 \%$ of intake ${ }^{4}$.

A recent Cochrane review investigated whether macronutrient interventions (such as high energy, high protein diets) influenced morbidity and mortality in adults and children living with HIV infection. Eight small randomised controlled trials (with a total of 486 participants) conducted in high-income countries were selected with the criteria based on evaluating the effectiveness of macronutrient interventions compared with no nutritional supplements or placebo. 
Overall, macronutrient supplementation (with or without nutritional counselling) significantly improved energy intake (5 trials; $\mathrm{n}=254$ ) and protein intake (3 trials; $\mathrm{n}=128$ ) compared with no nutritional supplementation or placebo. There was no evidence of an effect on body weight ( 8 trials; $n=423$ ), fat mass ( 6 trials; $n=305)$, fat-free mass (5 trials; $\mathrm{n}=311)$ or CD4 count (6 trials; $\mathrm{n}=271)^{61}$.

However positive outcomes of nutritional (macronutrient) intervention in patients with advanced AIDS wasting has been described in several non-controlled observation trials ${ }^{5}$.

In children, ready to use therapeutic foods (RUTFS) have been successful in treating severe malnutrition in non-HIV infected children. Studies suggest that in HIV infected children in food insecure areas that they can recover to normal nutritional status with normal protocols for severe acute malnutrition, but recovery can take longer than in nonHIV infected children ${ }^{62}$. Studies of the outcomes of using RUTFS in adults are limited.

\section{Food security interventions}

Few studies have assessed the outcomes of food supplementation in PLHIV in resource poor settings. One randomised trial of food support for food insecure PLHIV in Zambia, providing supplemental foods (not designed to meet total nutritional requirements) to patients receiving treatment demonstrated improvements in both nutritional status and adherence to ART. The study using multivariate analysis adjusting for sex, haemoglobin, and baseline CD4+ counts found significantly greater weight gain at 12 months, as well as better adherence among patients receiving food compared with those who did not receive food ${ }^{40}$.
Further studies are required to explore the effects of food support programs on weight gain, ART adherence, quality of life indicators, morbidity and mortality amongst food insecure PLHIV.

\section{CONCLUSION}

Most of the core principles of nutrition for people living with HIV are also the same for uninfected persons. A balanced healthy diet is essential for the health and survival of all people, regardless of HIV status. There is currently insufficient evidence to support an increase in protein, fat, or micronutrient requirements due to HIV infection per se. Micronutrients should be consumed at recommended daily intake levels.

Nutritional education should be an essential component of HIV related activities, as it can help PLHIV cope with symptoms of disease, prevent weight loss and manage side effects of medication. However it is important to remember that nutrition cannot cure HIV but is essential to maintain the immune system and help achieve optimal qualify of life.

In resource limited settings where wasting is one of the main concerns associated with lack of access to food, it is important to consider dietary diversity, as the majority of energy often comes from staples which may contain inadequate micronutrients. Government supported agricultural and social welfare policies are necessary to address deficiencies of household food security among individuals with HIV infection and their families.

The short and long-term benefits in terms of immune status, disease progression, physical function, and decreased morbidity and mortality resulting from nutritional interventions have not yet been proven. Further research in all aspects of HIV, nutrition and food security is required. 


\section{ACKNOWLEDGEMENTS}

The author would like to thank $\mathrm{Dr}$ Sarangapany Jeganathan, Head of Research at the Albion Street Centre, Sydney, Australia for reviewing this article and the Albion Street Centre Nutrition Development Division Dietitians for helping to build the evidence base for integrating nutrition in HIV care.

\section{REFERENCES}

1. UNAIDS, Report on the global AIDS epidemic; 2008.

2. UNAIDS/WHO Epidemiological Fact Sheets on HIV and AIDS, 2008 Update.

3. Mangili A, Murman DH, Zampini AM, Wanke CA. Nutrition and HIV infection: review of weight loss and wasting in the era of highly active antiretroviral therapy from the nutrition for healthy living cohort. Clin Infect Dis 2006; 42(6):836-42.

4. Hsu JW, Pencharz. Pencharz PB, Macallan. Macallan D, Tomkins A. Macronutrients and HIVIAIDS: A Review of Current Evidence. in Consultation on Nutrition and HIVIAIDS in Africa: Evidence, Lessons and Recommendations for Action. 2005. Durban, South Africa: WHO, Department of Nutrition for Health and Development.

5. Ockenga J, Grimble R, JonkersSchuitema C, Macallan D, Melchior J, Sauerwein $\mathrm{H}$ et al. ESPEN Guidelines on Enteral Nutrition: Wasting in HIV and other chronic infectious diseases. Clinical Nutrition 2006; 25, (2), 319-329.
6. Fields-Gardner C, Fergusson P, Position of the American Dietetic Association and Dietitians of Canada: nutrition intervention in the care of persons with human immunodeficiency virus infection. J Am Diet Assoc, 2004. 104(9):1425-41.

7. Kaiser J, Campa A, Ondercin J, Leoung $G$, Pless $R$, Baum $M$. Micronutrient supplementation increases CD4 count in HIVinfected individuals on Highly Active Antiretroviral therapy: A prospective double-blind, placebo-controlled trial. Journal of Acquired Immune Deficiency Syndrome 2006; 42(5):535-528.

8. Kotler DP, Wang, J, Pierson RN, Body composition studies in patients with the acquired immunodeficiency syndrome. Am J Clin Nutr, 1985. 42(6):1255-65.

9. Trujillo EB, Borlase BC, Bell SJ, Guenther KJ, Swails W, Queen PM, Trujillo JR. Assessment of nutritional status, nutrient intake, and nutrition support in AIDS patients. J Am Diet Assoc 1992; 92(4): 477-478.

10. Greene JB. Clinical approach to weight loss in the patient with HIV infection. Gastroenterol Clin North Am, 1988. 17(3). 573-86.

11. Scrimshaw NS, SanGiovanni JP. Synergism of nutrition, infection, and immunity: an overview. Am J Clin Nutr 1997; 66(2):464S-477S12.

12. Weiser SD, Leiter K, Bangsberg DR, Butler LM, Percy-de Korte $F$, et al. Food insufficiency is 
associated with high-risk sexual behavior among women in Botswana and Swaziland. PLoS Med 2007; 4: e260

13. Tang AM, Forrester J, Spiegelman D, Knox TA, Tchetgen E, Gorbach SL. Weight loss and survival in HIVpositive patients in the era of highly active antiretroviral therapy. J Acquir Immune Defic Syndr 2002; 31(2):230-6.

14. Severe $P$, Leger $P$, Charles $M$, Noel F, Bonhomme G, Bois G, et al. Antiretroviral therapy in a thousand patients with AIDS in Haiti. N Engl J Med 2005; 353 (22):2325-34.

15. Kotler DP, Tierney AR, Wang J, Pierson RN, Jr. Magnitude of body-cell-mass depletion and the timing of death from wasting in AIDS. Am J Clin Nutr 1989; 50(3):444-7.

16. Suttmann U, Ockenga J, Selberg O, Hoogestraat $L$, Deicher $\mathrm{H}$, Muller MJ. Incidence and prognostic value of malnutrition and wasting in human immunodeficiency virusinfected outpatients. J Acquir Immune Defic Syndr Hum Retrovirol 1995; 8(3):239-46.

17. Mocroft A, Kirk O, Barton SE, Dietrich $M$, Proenca $R$, Colebunders R, et al. Anaemia is an independent predictive marker for clinical prognosis in HIV-infected patients from across Europe. EuroSIDA study group. AIDS 1999; 13(8):94350.

18. Feldman JG, Gange SJ, Bacchetti $P$, Cohen M, Young
M, Squires KE, et al. Serum albumin is a powerful predictor of survival among HIV-1infected women. J Acquir Immune Defic Syndr 2003; 33(1):66-73.

19. van der Sande MA, Schim van der Loeff MF, Aveika AA, Sabally S, Togun T, Sarge-Njie $R$, et al. Body mass index at time of HIV diagnosis: a strong and independent predictor of survival. J Acquir Immune Defic Syndr 2004; 37(2):1288-94.

20. Macallan DC, Noble C, Baldwin C, Jebb SA, Prentice AM, Coward WA, et al. Energy expenditure and wasting in human immunodeficiency virus infection. N Engl J Med 1995; 333(2):83-8.

21. Arpadi SM, Cuff PA, Kotler DP, Wang J, Bamji M, Lange $M$, et al. Growth velocity, fat-free mass and energy intake are inversely related to viral load in HIV-infected children. J Nutr 2000; 130(10):2498-502.

22. Macallan DC. Wasting in HIV infection and AIDS. J Nutr 1999; 129(1S Suppl):238S-242S.

23. Griffin GE. Malabsorption, malnutrition and HIV disease. Baillieres Clin Gastroenterol 1990; 4(2):361-73.

24. Macallan DC, Griffin GE. Metabolic disturbances in AIDS. $N$ Engl J Med 1992; 327(21):1530-1.

25. Amadi B, Kelly P, Mwiya $M$, Mulwazi E, Sianongo S, Changwe $\mathrm{F}$, et al. Intestinal and systemic infection, HIV, and 
mortality in Zambian children with persistent diarrhoea and malnutrition. J Pediatr Gastroenterol Nutr 2001; 32(5):550-4.

26. Macallan DC, Noble C, Baldwin C, Foskett M, McManus $T$, Griffin GE. Prospective analysis of patterns of weight change in stage IV human immunodeficiency virus infection. Am J Clin Nutr 1993; 58(3):417-24.

27. Keating J, Bjarnason I, Somasundaram $S$, Macpherson $A$, Francis $N$, Price $A B$, et al. Intestinal absorptive capacity, intestinal permeability and jejunal histology in HIV and their relation to diarrhoea. Gut 1995; 37(5):623-9.

28. Hommes MJ, Romijn JA, Godfried MH, Schattenkerk JK, Buurman WA, Endert E, et al. Increased resting energy expenditure in human immunodeficiency virus-infected men. Metabolism 1990; 39(11):1186-90.

29. Hommes MJ, Romijn JA, Endert E, Sauerwein HP. Resting energy expenditure and substrate oxidation in human immunodeficiency virus (HIV)infected asymptomatic men: HIV affects host metabolism in the early asymptomatic stage. Am J Clin Nutr 1991; 54(2):3115.

30. Grunfeld C, Pang M, Shimizu L, Shigenaga JK, Jensen $P$, Feingold KR. Resting energy expenditure, caloric intake, and short-term weight change in human immunodeficiency virus infection and the acquired immunodeficiency syndrome. Am J Clin Nutr 1992; 55(2):45560.

31. Melchior JC, Salmon D, Rigaud D, Leport C, Bouvet E, Detruchis $P$, et al. Resting energy expenditure is increased in stable, malnourished HIVinfected patients. Am J Clin Nutr 1991; 53(2):437-41.

32. Melchior JC, Raguin G, Boulier $A$, Bouvet $E$, Rigaud $D$, Matheron $S$, et al. Resting energy expenditure in human immunodeficiency virus-infected patients: comparison between patients with and without secondary infections. Am J Clin Nutr 1993; 57(5):614-9.

33. FAO. Incorporating HIVIAIDS Considerations into Food Security and Livelihood Projects. FAO: Rome. 2003, 45.

34. Lemke S. Nutrition security, livelihoods and HIVIAIDS: implications for research among farm worker households in South Africa. Public Health Nutr, 2005. 8(7): p. 844-52.

35. Stover and Bollinger. The Economic impact of AIDS. 1999, The Policy Project.

36. Raiten D, Grinspoon S, Arpadi $S$. Nutritional considerations in the use of ART in resourcelimited settings. Geneva: World Health Organization; 2005.

37. Castleman T, Seumo E, Cogill $B$, Food and Nutrition Implications of Antiretroviral Therapy in Resource Limited Settings. 2003, Food and 
Nutrition Technical Advisory Group: Washington

38. Paton N, Sangeetha S, Earnest $A$, Bellamy R. The impact of malnutrition on survival and the CD4 count response in HIVinfected patients starting antiretroviral therapy. HIV Medicine 2006; (7):323-330.

39. Kaiser J, Campa A, Ondercin J, Leoung $\mathrm{G}$, Pless $\mathrm{R}$, Baum $\mathrm{M}$. Micronutrient supplementation increases CD4 count in HIVinfected individuals on Highly Active Antiretroviral therapy: A prospective double-blind, placebo-controlled trial. Journal of Acquired Immune Deficiency Syndrome 2006; 42(5):535-528.

40. Megazzini KS, Washington $M$, Sinkala M, Lawson-Marriot $S$, Stringer E, Krebs D, et al . A pilot randomised trial of nutritional supplements in food insecure patients receiving antiretroviral therapy in Zambia. In: Sixteenth International AIDS Conference; 2006; Toronto, Canada; 2006.

41. Chlebowski RT, Grosvenor $M$, .Lillington L, Sayre J. Beall G. Dietary intake and counseling, weight maintenance, and the course of HIV infection. J Am Diet Assoc, 1995. 95(4): 42832.

42. van Niekerk C, Smego R, Sanne I. Effect of nutritional education and dietary counselling on body weight in HIV-seropositive South Africans not receiving antiretroviral therapy. J Hu Nutr Dietet, 2000. 13: 407-412.
43. World Health Organisation. Health Topic. Diarrhoea. Available at http://www.who.int/topics/diarrh oea/en/. Accessed 07/05/09

44. Anastasi JK, Capili B, Kim A G, McMahon D, Heitkemper M. M. 'Symptom management of HIVrelated diarrhea by using normal foods: A randomized controlled clinical trial'. Journal of the Association of Nurses in AIDS Care. 2006:17(2):47-57.

45. Sifri R, Diaz VA, Gordon L, Glick M, Anapol H, Goldschmidt $\mathrm{R}$, et al. Oral health care issues in HIV disease: developing a core curriculum for primary care physicians. The Journal of the American Board of Family Practice, 1998, 11, (6) 434-444.

46. Adetifa I, Okomo U. Iron supplementation for reducing morbidity and mortality in children with HIV. Cochrane Database of Systematic Reviews 2009, Issue 1. Art. No.:CD006736.

47. Fawzi W, Msamanga GI, Spiegelman D, Wei $R$, Kapiga $S$, Villamor $E$, et al. A randomized trial of multivitamin supplements and HIV disease progression and mortality. $\mathrm{N}$ Engl J Med 2004, 351(1): 2332.

48. Jiamton S, Pepin J, Suttent R, Filteau S, Mahakkanukrauh B, Hanshaoworakul W, et al. A randomized trial of the impact of multiple micronutrient supplementation on mortality among HIV-infected individuals living in Bangkok. Aids 2003; 17(17):2461-9. 
49. Abrams B., D. Duncan, and I. Hertz-Picciotto. A prospective study of dietary intake and acquired immune deficiency syndrome in HIV-seropositive homosexual men. $J$ Acquir Immune Defic Syndr 1993, 6(8): 949-58.

50. Tang AM, Graham NM, Kirby AJ, McCall LD, Willett WC, Saah AJ. Dietary micronutrient intake and risk of progression to acquired immunodeficiency syndrome (AIDS) in human immunodeficiency virus type 1 (HIV-1)-infected homosexual men. Am J Epidemiol 1993; 138(11):937-51.

51. Tang AM, Graham NM, Saah AJ. Effects of micronutrient intake on survival in human immunodeficiency virus type 1 infection. Am J Epidemiol 1996; 143(12):1244-56.

52. Kanter AS, Spencer DC, Steinberg $M H$, Soltysik $R$, Yarnold PR, Graham NM. Supplemental vitamin $B$ and progression to AIDS and death in black South African patients infected with HIV. J Acquir Immune Defic Syndr 1999; 21(3):252-3.

53. Tang AM, Graham NM, Semba,R. D, Saah A. J. Association between serum vitamin $A$ and $E$ levels and HIV1 disease progression. 1997 AIDS 11 (5): 613-20.

54. Villamor E, Saathoff E, Bosch RJ, Hertzmark E, Baylin A, Manji K, et al. Vitamin supplementation of HIV-infected women improves postnatal child growth. Am J Clin Nutr 2005; 81(4):880-8.

55. Bobat R, Coovadia H, Stephen C, Naidoo KL, McKerrow N, Black RE, et al. Safety and efficacy of zinc supplementation for children with HIV-1 infection in South Africa: a randomised double-blind placebo-controlled trial. Lancet 2005, 366(9500): 1862-67.

56. Coutsoudis A, Bobat RA, Coovadia HM, Kuhn L, Tsai WY, Stein ZA. The effects of vitamin $A$ supplementation on the morbidity of children born to HIV-infected women. $A m \mathrm{~J}$ Public Health 1995, 85(8 pt. 1): 1076-81.

57. Fawzi W, Mbise RL, Hertzmark E, Fataki MR, Herrera MG, Ndossi $G$, et al. A randomized trial of vitamin A supplements in relation to mortality among human immunodeficiency virusinfected and uninfected children in Tanzania. Pediatr Infect Dis $J$ 1999, 18(2): 127-33.

58. Drain K, Kupka R, Mugusi F, Fawzi. Micronutrients in HIVpositive persons receiving highly active antiretroviral therapy. American Journal of Clinical Nutrition, 200785 (2), 333-345.

59. Friis $\mathrm{H}$. Micronutrients and HIV infection: a review of current evidence: Consultation on Nutrition and HIVIAIDS in Africa: Evidence, lessons and recommendations for action. Durban, South Africa: World Health Organization; 2005. 
60. Mannheimer S, Friedland G, Matts J, Child C, Chesney M. The consistency of adherence to antiretroviral therapy predicts biologic outcomes for human immunodeficiency virus-infected persons in clinical trials. Clin Infect Dis 2002; 34(8):1115-21.

61. Mahlungulu S, Grobler LA, Visser ME, Volmink J. Nutritional interventions for reducing morbidity and mortality in people with HIV. Cochrane
Database of Systematic Reviews 2007, Issue 3. Art. No.: CD004536.

62. Diop EH, Dossou NI, Ndour $M M$, Briend $A$, Wade $S$. Comparison of efficacy of a solid ready-to use food and a liquid, milk-based diet for the rehabilitation of severely malnourished children: randomized trial Am J Clin Nutr 2003; 78: 302-7. 